\title{
Development of an Illumination Control Method to Improve Office Productivity
}

\author{
Fumiaki Obayashi, Misa \\ Kawauchi, Masaaki Terano \\ Matsushita Electric Works, Ltd., 1048 \\ Kadoma, Kadoma, Osaka, Japan \\ \{obayashi, kawauchi.misa, \\ terano\}@mail.mew.co.jp
}

\author{
Kazuhiro Tomita, Yoko Hattori, Hiroshi \\ Shimoda, Hirotake Ishii, Hidekazu Yoshikawa \\ Graduate School of Energy Science, Kyoto \\ University Gokashou, Uji, Kyoto, Japan \\ \{t-kazuhiro, h-yoko, shimoda, hirotake, \\ yosikawa\}@energy.kyoto-u.ac.jp
}

\begin{abstract}
A lighting control method has been proposed which adjusts the circadian rhythms of office workers and eliminates a decrease in the arousal level in the afternoon by exposing a high intensity of light in the morning in order to improve their productivity. As a result, it was found that the task performance of the CPTOP test under 2,100 lx was significantly higher than that under $700 \mathrm{~lx}$ and more than a 9\% improvement of task performance was achieved. Furthermore, a demonstration experiment has been conducted in order to confirm the effectiveness of the proposed lighting control method in actual office rooms. As a result, it was confirmed that the performance of their accounting work was over $10 \%$ higher than under normal conditions.
\end{abstract}

\section{Introduction}

A lot of people are spending considerable time within a day in the office and in public places, ex. libraries, and the approach on health and the comfort there is very important from the aspect of total QOL (Quality of Life). Health and the comfort of residents, however, it is generally indefinite cost-effectiveness, and this becomes a barrier to introduce equipment for residents. Therefore, the appeal power from which such "Health and Comfort for residents" technology is widely introduced into the office and public places are required. Then, the study where the index on the end user side (Health and Comfort) is caught by the index on the management side of clarification of cost-effectiveness again thus is being promoted.

So, productivity of office workers has become a new evaluation viewpoint of the office environment. Increase of office productivity by indoor environmental improvement produces great economic advantages. A trial calculation [1] shows the economic value of office productivity being $\$ 58,508$ /year in case productivity has improved by $3 \%$ in a 10,000-square foot office building. Office productivity is a new index to produce a better indoor environment for office workers, and to increase building value. A method which can evaluate office work productivity objectively and quantitatively has been developed [2]. 
In this study, therefore, the lighting control method for the office has been developed and a basic experiment has been conducted to evaluate how much office work productivity is improved by controlling lighting conditions in the office space.

\section{Office Productivity Index and Evaluation Method}

Various definitions and interpretations of office productivity have been suggested. In this study, Office Productivity is defined as office working efficiency.

In order to calculate office productivity of a specified occupation category, the results of the performances of the elemental abilities are given added weight to each performance based on the deduced weight. The concrete productivity index can be calculated by equation (1).

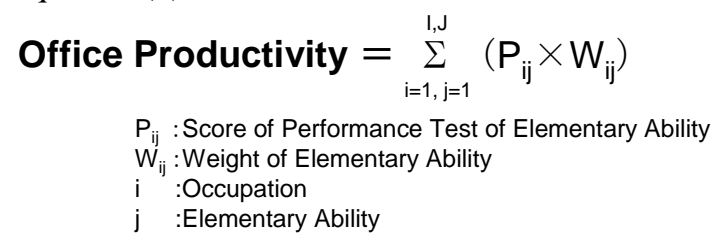

This index can be calculated by the results of performance tests and weights of elementary abilities for a specific occupation and a specific office environment.

A performance test system, CPTOP (Cognitive Performance Tests for Office Productivity) has been developed to evaluate the productivity of office workers objectively and quantitatively based on the above productivity evaluation conception [2]. CPTOP is a web-based performance test system and it can be used by only PCs and Web browsers connected to the Internet as shown in Figure 1.

The CPTOP consists of 11 different task tests which correspond to 11 elementary abilities. By changing the configuration of the test set and weights, productivity in various office situations and environments can be evaluated. The results of the performance tests are dependent on individual abilities, the statistical calculation among plural subjects or comparison of the same subject before and after changing the environment is necessary to evaluate the office environment precisely. Note that this test is to evaluate productivity in a specific office environment, and NOT to evaluate the abilities of individual workers.

CPTOP

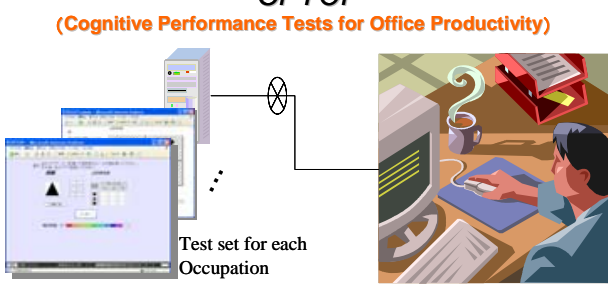

Fig. 1. Configuration of CPTOP

In order to evaluate any improvements of the office environment by using this method, productivity index before and after improvement is compared. 


\section{Productivity and Office Lighting}

\subsection{Design of lighting environment}

Present office lighting is designed from the viewpoints of eye sight and stress reduction for the optic nerve, and it is considered as the minimum requirement for the office environment. In order to make the appropriate effect for productivity by lighting conditions, a lighting control method has been designed.

From the viewpoint of adjusting the human circadian rhythm, it is necessary to be exposed to thousands of lux lights in the morning for a few hours. It is known that exposure to a high intensity of illumination adjusts the human circadian rhythm and the arousal level can be increased as the intensity soars up to $10,000 \mathrm{~lx}$ [3]. In addition, if the circadian rhythm is synchronized with life rhythm and in keeping with a high arousal level in the day time, a high quality of sleep can be obtained. On the contrary, a high quality of sleep at the night causes higher arousal levels in the day time. This means that high illumination light keeps high arousal levels in the office. However from the standpoint of productivity in the long term, it is not appropriate because keeping high arousal levels all day may cause workers' fatigue. Therefore, the high illumination light should be applied only to regulate biological rhythm and improve the arousal level.

When aiming at adjusting biological rhythm, improvement of arousal level and reduction of long term fatigue at the same time, the lighting control method as shown in Figure 2 was proposed. It compensates for a lack of light by giving high illumination light in the morning and adjusts the biological rhythm by resetting the biological clock. In the early afternoon, high illumination light is increases to reduce sleepiness after lunch. After that the illumination decreases to $700 \mathrm{~lx}$, which is the illumination of a normal office. During lunch time, the illumination is decreased to $500 \mathrm{~lx}$ to reduce energy consumption and promotes taking a nap to reduce sleepiness after lunch.

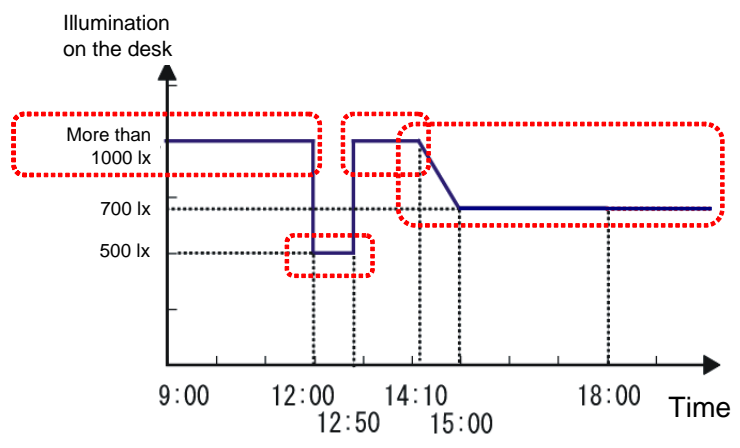

Fig. 2. Proposed lighting control method

It is expected that the lighting control method can reduce long term fatigue by adjusting biological rhythm and improve the work performance in the short term by keeping high arousal level and preventing from sleepiness after lunch. 


\subsection{Productivity evaluation of proposed lighting control method}

In order to evaluate effectiveness of the proposed lighting control method from the viewpoint of productivity, a subjective experiment was conducted to quantitatively evaluate it by using CPTOP. At the same time, a proper lighting control method was studied by comparing different maximum illumination of the lighting control pattern.

\subsubsection{Experimental method}

Based on the control pattern as shown in Figure 2, two conditions in which the maximum illuminations are 1,400 lx (condition 1) and 2,100 lx (condition 2) were prepared. In addition, normal condition in which the illumination is fixed to $700 \mathrm{~lx}$ (normal condition) was also prepared.

The subjects of the experiment were 6 people with experience in office work. Their average age was 27.7 (from 22 to 36) and they were accustomed to using a PC. The experiment was conducted in an experiment room where the lighting condition can be controlled. Figure 3 shows the top view of the experimental room, while Figure 4 shows a view of the experiment. The lighting equipment were 12 illumination panels and 8 ceiling lights, which can give 500 to 3,000 lx illumination. Figure 5 shows the actual illumination measured on the surface of the desk by an illumination meter.

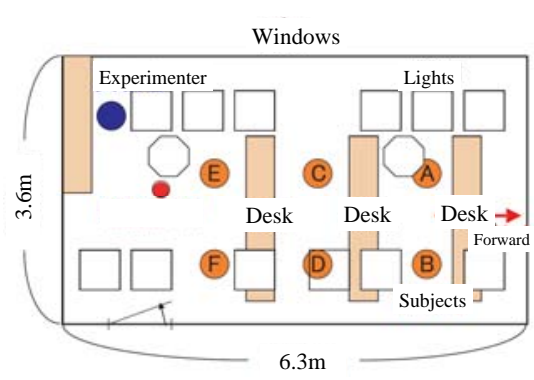

Fig. 3. Top view of experimental room

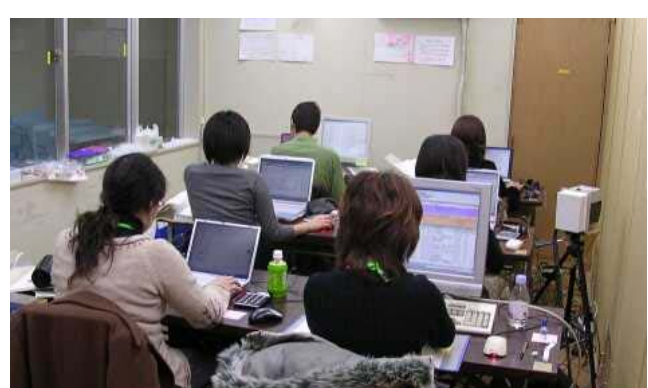

Fig. 4. A scene of experiment

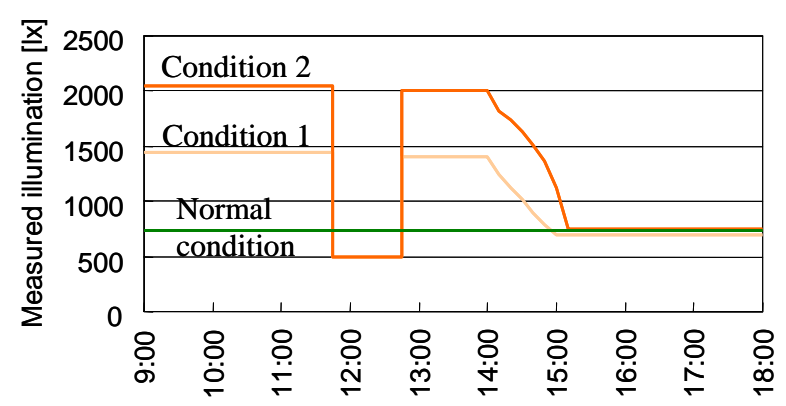

Fig. 5. Measured illumination

In order to evaluate the effectiveness of the lighting control method, CPTOP was conducted as a quantitative evaluation. In addition, a questionnaire was also 
conducted to evaluate subjective fatigue by using an examination sheet for subjective symptoms provided by the Japan Society for Occupational Health [4].

The experiment was conducted for 14 days along with the schedule as shown in Figure 6. And the experimental procedure of a day is shown in Figure 7. One day is divided into three parts morning, early afternoon and late afternoon. In each part, the questionnaire was conducted first, and simulated office tasks were given, then the CPTOP was conducted. The time for CPTOP was controlled to approximately 60 minute time lots. In each condition except for the first day and the fourth day, simulated office tasks were given instead of CPTOP as just a task workload.

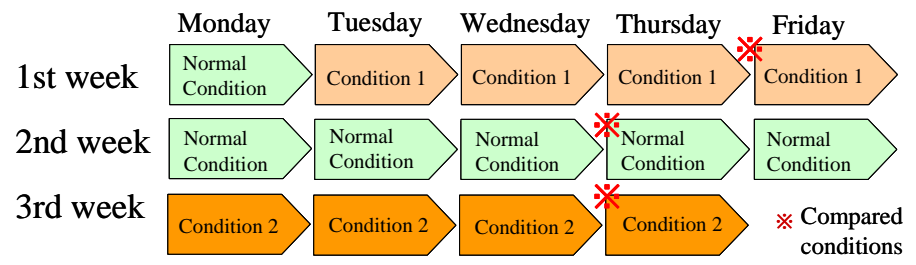

Fig. 6. Schedule of experiment.

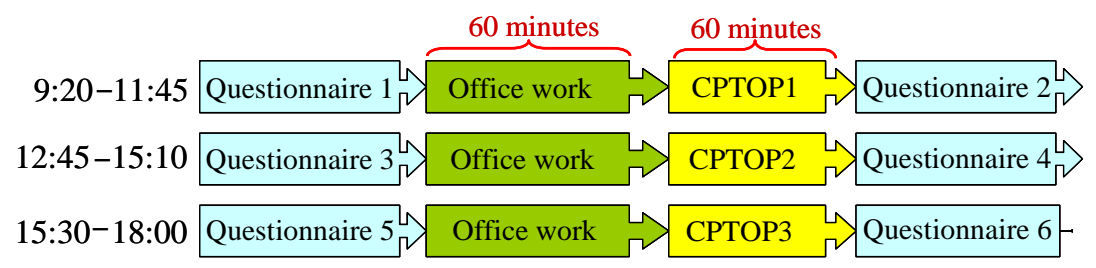

Fig. 7. Experimental Procedure.

\subsubsection{Result}

Figure 8 shows the average score of CPTOP of all the subjects. Here, the scores were standardized based on the average score of all the experimental terms for each subject, and they were calculated as weights of the 11 task tests which were assumed to be equal.

As a result of CPTOP, the scores of condition 2 are significantly higher than those of normal conditions. Among condition 2, the scores of the third day and the fourth day are higher than that of the first day $(\mathrm{p}<.05$ and $\mathrm{p}<.01$, respectively). This means that the task performance was improved by the high illumination light. On the other hand, there is no difference between condition 1 and normal conditions. This means that the illumination of 1,400 lx is not bright enough to improve the performance.

Figure 9 shows the results of the questionnaire. As shown in Figure 12, total score of subjective fatigue in three days of condition 2 except for the third day which is significantly lower than those in normal conditions. It is presumed that illumination condition may affect subjective fatigue. 


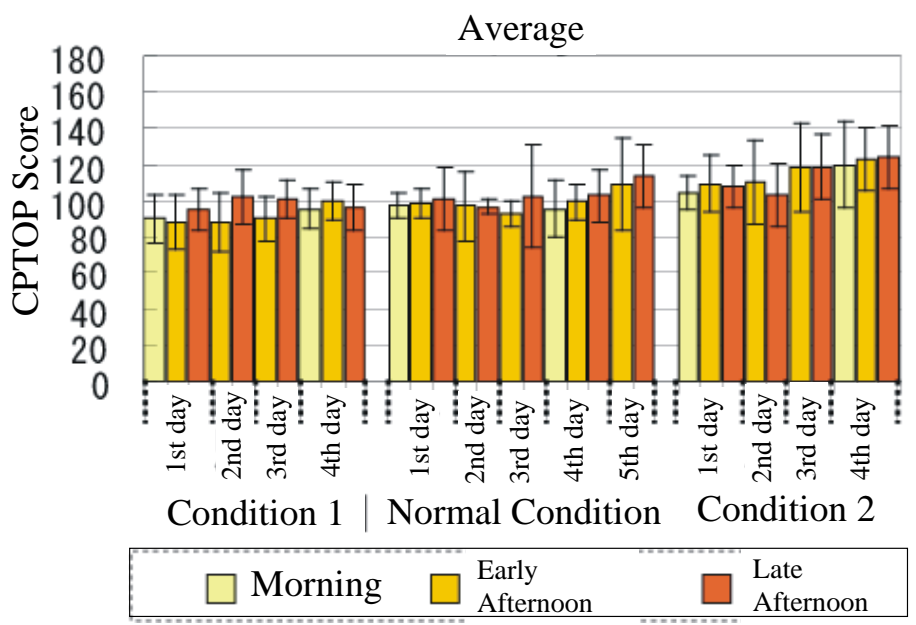

Fig. 8. Average score of CPTOP of all the subjects.

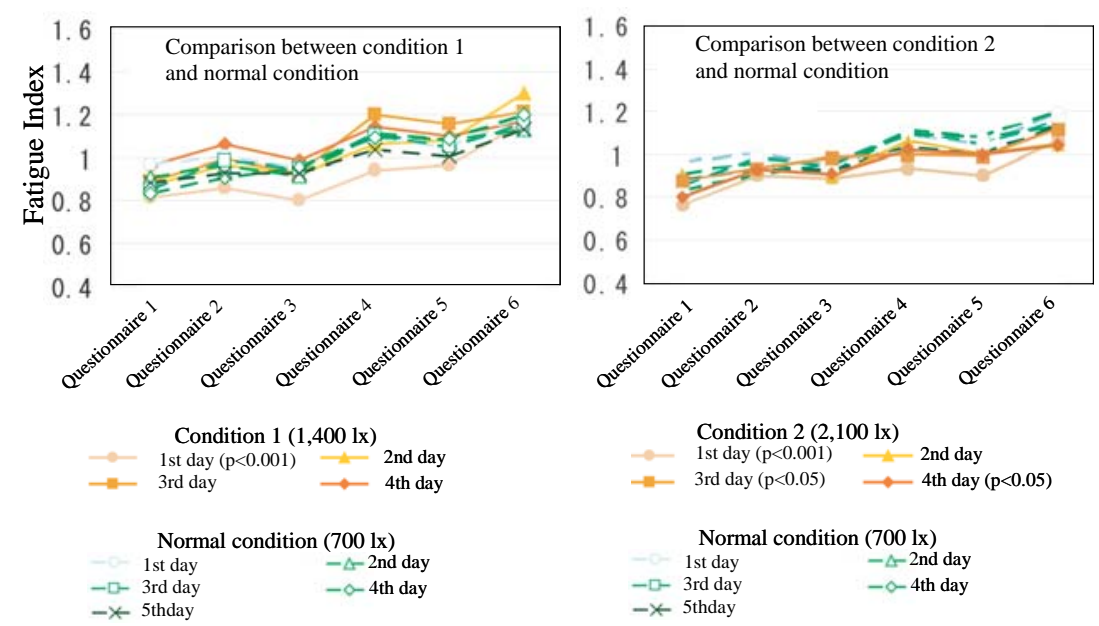

Fig. 9. Results of questionnaire for subjective fatigue.

\subsubsection{Evaluation of productivity}

The purpose of this experiment is the quantitative evaluation of productivity by the proposed lighting control method. The score of CPTOP in the fourth day of condition 2 and normal condition were compared statistically. As a result, the score of condition 2 is 9 points higher than that of normal condition $(\mathrm{p}<.001)$. The score of 
CPTOP is standardized to 100 , therefore a $9 \%$ improvement of task performance can be expected when controlling lighting along with Figure 2 with 2,100 lx for four days.

\section{Office Demonstration Experiment of Productivity Lighting System}

In order to confirm the effectiveness of the proposed lighting control method, a demonstration experiment in an actual office has been conducted.

\subsection{Experimental method}

Based on the control pattern as shown in Figure 2, one condition in which the maximum illumination is 3,500 lx (condition 3) was prepared. Also, normal conditions in which the illumination is fixed to $700 \mathrm{~lx}$ (normal condition) was prepared.

The subjects of the experiment were 6 people who worked in the accounting department of a company. The experiment was conducted in their office room where the additional light fitting was set up. Figure 10 shows the top view of the desks where subjects use, while Figure 11 shows a view of the office. The light equipments can give 300 to $4,000 \mathrm{~lx}$ illumination. Figure 12 shows the actual illumination measured on the surface of each desk by an illumination meter.

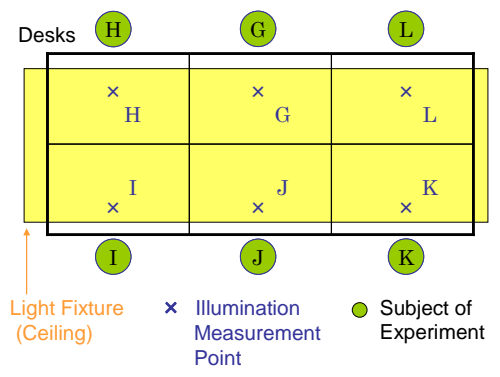

Fig. 10. Top view of experimental place

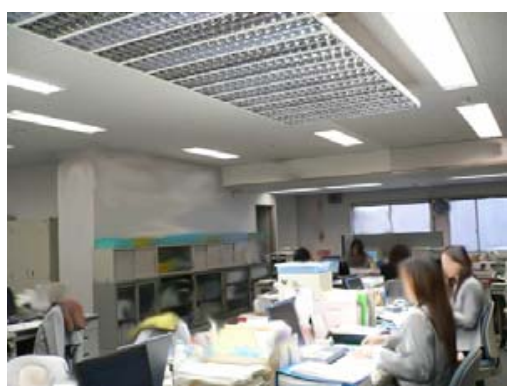

Fig. 11. A scene of office

In order to evaluate the effectiveness of the lighting control method, the office workers quantity of work is measured. The subjects of the experiment elected one preferred hour in each day. The number of sheets of the slip processing in the accounting processing work is measured. The number of sheets a minute is assumed to be an amount of real work. The accounting processing works by extracting necessary data from the slip of paper, then recording the electronic data, and finally checking the correspondence of the slip. The experiment was conducted from late November to early January. The subjects of the experiment selected days which were an average amount of work for this period, and the measurement was conducted. The 
lighting condition 3 is conducted in one week in mid-December. About three weeks are made for the experiment period of a standard condition before and after. Each subject selected three days as measurement days from each period.

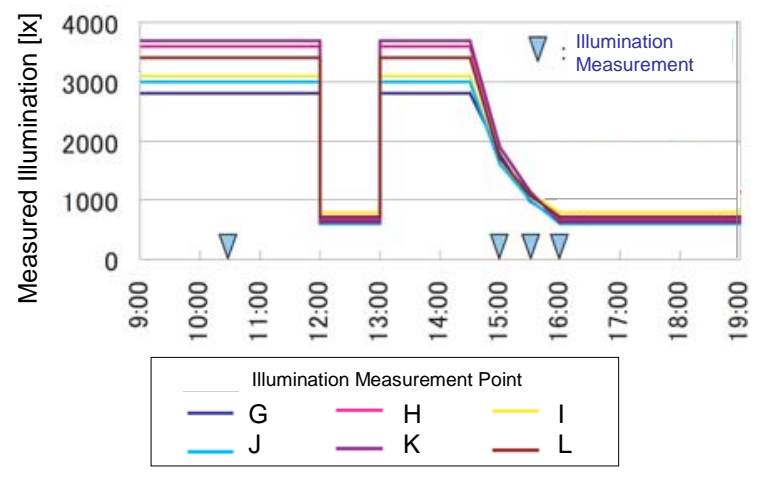

Fig. 12. Measured illumination

\subsection{Evaluation}

Figure 13 shows the performance of work in the each subject's accounting process work. For four of six subjects, the amount of work under the lighting condition 3 is higher than the amount of work under the standard conditions which were conducted before and after the lighting condition 3.

As a result, it is shown that their performances have improved with the lighting system. About a ten percent or over improvement of work performance can be expected when controlling lighting along with Figure 2.

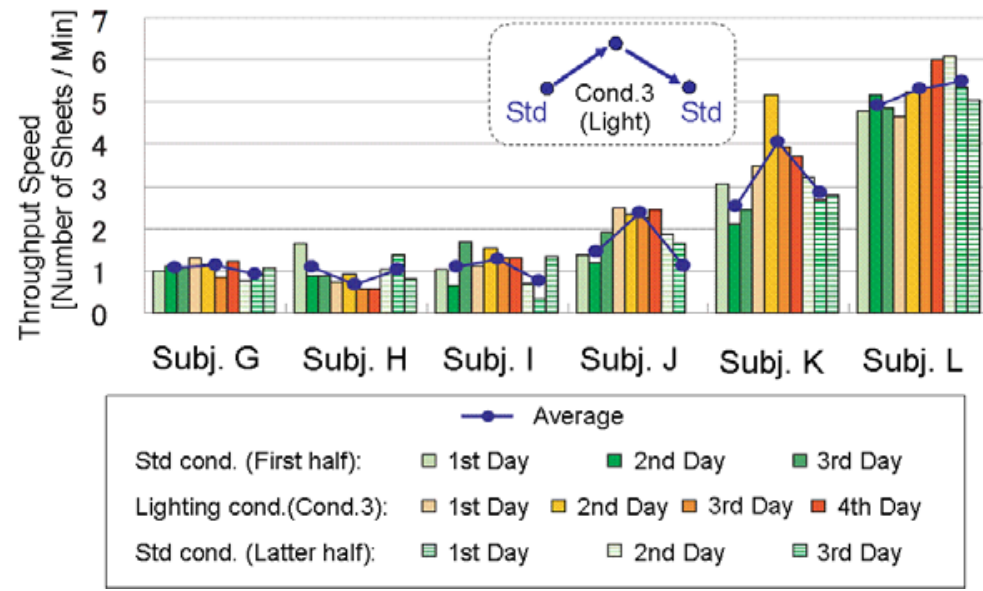

Fig. 13. Performance of Work in Each Subject's Accounting Processing Work 
Table 1. Comparison of Performance between Lighting Control Condition and Standard Condition

\begin{tabular}{|c|c|c|}
\hline $\begin{array}{l}\text { Lighting / Std (First half) } \\
\text { (Cond. 3) }\end{array}$ & $\begin{array}{c}\text { Lighting / Std (Latter half) } \\
\text { (Cond. 3) }\end{array}$ & $\begin{array}{c}\text { Lighting / Std (Average) } \\
\text { (Cond. 3) }\end{array}$ \\
\hline 1.180 & 1.211 & 1.184 \\
\hline
\end{tabular}

\section{Conclusions}

In this study, the lighting control method has been proposed which improves productivity of office workers and adjusts their circadian rhythms. A subjective experiment was conducted to evaluate a proposed lighting control method which can adjust circadian rhythm of office workers by exposing them to high illumination light. In the experiment, three lighting conditions were prepared which were normal condition (700 lx fixed), condition 1 (maximum illumination was 1,400 lx and control pattern was along with Figure 2) and condition 2 (2,100 lx as well). Under these lighting conditions, CPTOP and questionnaires for subjective fatigue were conducted. As a result, it was found that the CPTOP score under the condition 2 was $9 \%$ higher than that under the normal condition, and subjective fatigue under condition 2 was significantly lower than that of under normal conditions.

Furthermore, a demonstration experiment has been conducted in order to confirm the effectiveness of the proposed lighting control method in actual office rooms. As a result, it was confirmed that the performance of their accounting work was over $10 \%$ higher than that of under normal conditions.

In the future, we will confirm the effectiveness of the proposed lighting control method in various actual office rooms.

\section{References}

[1] Lomonaco, G., et al. (1997). Environmental Satisfaction, Personal Control and Positive Correlation to Increased Productivity, Johnson Controls.

[2] Obayashi, F., Kawauchi, M., Terano, M., Ito, K., Shimoda, H. and Yoshikawa, H.(2005) Objective Measurement Method for Productivity and Investigation on Environmental Factor, Proceedings of Human Computer Interaction International 2005, CD-ROM

[3] NEMA (1989) Lighting and Human Performance: A Review, Lighting Equipment Division of National Electrical Manufactures Association and the Lighting Research Institute.

[4] Japan Society of Occupational Health (1988) Handbook of Industrial Fatigue, Roudou Chousakai (in Japanese). 